$\begin{array}{cc}\text { Programa de Pós-Graduação em Engenharia de Produção - PPGEP } & \\ \text { Laboratório de Qualidade de Vida - LaQVida } & \text { REVISTA BRASILEIRA DE } \\ \text { Universidade Tecnológica Federal do Paraná - UTFPR } & \text { Ponta Grossa - PR - Brasil } \\ \text { v. 06, n. 02, abr./jun. 2014, p. 66-76 } & \text { QUALIDADE DE VIDA }\end{array}$

DOI: $10.3895 / \mathrm{S} 2175-08582014000200001$

\title{
Revisão da literatura sobre o estilo de vida de estudantes universitários
}

\section{Literature review on the lifestyle of college students}

\author{
Bruno José de Queiroz Brito \\ Universidade Federal do Recôncavo da Bahia - UFRB - Amargosa - Bahia - Brasil \\ brunoqueiroz.e.d.f@gmail.com \\ Alex Pinheiro Gordia \\ Universidade Federal do Recôncavo da Bahia - UFRB - Amargosa - Bahia - Brasil \\ alexgordia@gmail.com \\ Teresa Maria Bianchini de Quadros \\ Universidade Federal do Recôncavo da Bahia - UFRB - Amargosa - Bahia - Brasil \\ tetemb@gmail.com
}

\section{RESUMO}

OBJETIVO: O presente artigo teve como objetivo revisar a literatura referente ao estilo de vida (EV), em especial sobre suas definições, instrumentos de avaliação, bem como, apresentar achados de estudos nacionais e internacionais sobre o EV de estudantes universitários.

MÉTODOS: Foi realizada uma busca de trabalhos em bases de dados eletrônicas (PUBMED, LILACS, SCIELO) e no site de busca Google Acadêmico, utilizando descritores acerca do tema em português, inglês e espanhol.

RESULTADOS: No que se refere à definição do EV, embora o conceito desenvolvido pela Organização Mundial da Saúde (OMS) seja bastante aceito, ainda não há um consenso na literatura sobre qual definição melhor o representa. Quanto à avaliação do EV, constatou-se aumento considerável nos últimos anos na quantidade de instrumentos, bem como, observou-se que estes instrumentos estão sendo desenvolvidos, traduzidos e validados em diversos países. No que se refere ao EV de estudantes universitários, embora haja relevantes diferenças regionais, notou-se que em grande parte dos estudos os resultados foram preocupantes, pois indicaram adesão a um EV pouco saudável, com elevado consumo de álcool e tabaco, baixo nível de atividade física e hábitos alimentares inadequados.

CONCLUSÕES: Os achados do presente estudo indicaram relevante avanço do conhecimento sobre o EV durante os últimos anos. Contudo, grande parte dos estudantes universitários tem adotado um EV pouco saudável. Neste sentido, observa-se a necessidade da elaboração e implantação de políticas públicas de promoção da saúde dentro da própria Universidade, com vistas à melhoria da saúde e da qualidade de vida dos estudantes universitários.

PALAVRAS-CHAVE: Estilo de vida. Estudantes universitários. Qualidade de vida. 


\section{ABSTRACT}

OBJECTIVE: This article aims to review the literature related to lifestyle (EV), in particular on their definitions, assessment tools, as well as presenting findings in national and international studies on the EV of college students.

METHODS: A search of work was performed in electronic databases (PubMed, LILACS, SciELO) and search Google Scholar site using descriptors on the subject in English, Portuguese and Spanish. RESULTS: As regards the definition of the EV, although the concept developed by the World Health Organization is widely accepted, there is still no consensus in the literature about which is the best definition. Regarding the evaluation of EV, considerable increase in recent years in the number of instruments is found, and it was noted that these instruments are being developed, translated and validated in several countries. With regard to the EV of college students, although there are significant regional differences, it was noted that in most studies the results were worrying because indicated adherence to an unhealthy EV, with high consumption of alcohol and tobacco, low physical activity and poor dietary habits.

CONCLUSIONS: The findings of this study indicated significant advancement of knowledge about the EV over the last years. However, most college students have adopted an unhealthy EV. In this sense, we observe the need of elaboration and implementation of public policies to promote health within the University itself, with a view to improving the health and quality of life of college students.

KEYWORDS: Lifestyle. College students. Quality of life.

\section{Introdução}

O impacto dos hábitos pessoais e do estilo de vida (EV) sobre a saúde das pessoas está bem documentado na literatura (BLAIR et al., 1996; CERVATO et al., 1997; MOTA; DUARTE, 1999; RICHARDSON et al., 2004; VILARTA; GONÇALVES, 2004). Ainda que a genética, o ambiente e a assistência médica tenham um papel importante em como e quanto se vive, cada vez mais crescem as evidências de que o modo de viver representa diferencial para a saúde e a qualidade de vida no mundo contemporâneo, independente da idade ou condição social (NAHAS; BARROS; FRANCALACCI, 2000).

O EV é um dos fatores preponderantes para a manutenção, tanto da qualidade de vida quanto da saúde das pessoas, revelando que esta tríade (EV, qualidade de vida e saúde) está intimamente associada ao bem-estar (ASTRAND, 1993; HEYWARD, 1998; MOTA; DUARTE, 1999; RICHARDSON et al., 2004; VILARTA; GONÇALVES, 2004). Um EV conduzido por hábitos saudáveis é necessário para a manutenção da saúde física, mental e espiritual da pessoa, sendo um dos aspectos que colaboram para uma satisfatória qualidade de vida (SOARES; CAMPOS, 2008).

Existem fatores do EV que afetam de forma negativa a saúde, e sobre os quais se pode ter controle, chamados de fatores negativos modificáveis. Entre estes fatores, pode-se citar, por exemplo, o fumo, o álcool, as drogas, o estresse e a falta de atividade física. Há ainda os fatores positivos, que se ministrados de forma correta, contribuem também para um EV mais saudável, como a alimentação saudável, a prática de atividade física e os comportamentos preventivos (NAHAS; BARROS; FRANCALACCI, 2000).

Considerando as situações próprias da adolescência e do adulto jovem, com significativas instabilidades biopsicossociais, o ingresso no meio universitário proporciona novas relações sociais e adoção de novos comportamentos, tornando os indivíduos vulneráveis às condutas de risco à saúde (VIEIRA et al., 2002). Dessa forma, a investigação do EV de estudantes universitários é de suma importância para verificar a necessidade de implantação de políticas de saúde coletiva nos campi universitários, visando à modificação de um EV considerado pouco saudável por parte dos 
acadêmicos. Contudo, ainda que a preocupação com o EV de estudantes universitários tenha aumentado nos últimos anos, estudos que tenham sistematizado estes achados são escassos, mesmo em teses ou dissertações e revisões da literatura sobre a temática são raras (MARINHO, 2002).

A revisão da literatura do presente estudo foi realizada nas bases de dados eletrônicas SciELO, MEDLINE/PubMed, LILACS e no site de busca Google Acadêmico, através de pesquisa manual em periódicos brasileiros não indexados, de busca específica por autores e em livros clássicos da área. Foram utilizados os seguintes descritores, de forma isolada e combinada, em língua portuguesa e sua correspondência em inglês e espanhol: 'estilo de vida' e 'universitários'. Não foi determinado um período de tempo específico de publicação para a busca e utilização de trabalhos na revisão, considerando tanto artigos originais quanto de revisão. Foram localizados 10.294 artigos, sendo que foram analisados e utilizados na presente revisão apenas os trabalhos que tratavam sobre a temática EV em universitários, totalizando 35 estudos. Trabalhos que tinham apenas o resumo disponível impossibilitando a leitura do manuscrito na íntegra não foram utilizados.

O presente artigo teve como objetivo revisar estudos referentes ao $\mathrm{EV}$, em especial sobre suas definições, instrumentos de avaliação, bem como apresentar achados de estudos nacionais e internacionais sobre o EV de estudantes universitários.

\section{Estilo de vida de estudantes universitários}

\subsection{Estilo de vida: definições e conceitos}

O EV é caracterizado por padrões de comportamento identificáveis que podem ter efeito profundo na saúde dos seres humanos, além de estar relacionado com diversos aspectos que refletem as atitudes, os valores e as oportunidades na vida das pessoas (WHO, 1998).

$\mathrm{Na}$ literatura podem-se encontrar vários conceitos acerca do EV. Sorokin (1947 apud GONÇALVES, 2004, p. 3) definiu o EV como sendo "[...] tudo o que o indivíduo aprende a fazer para viver numa comunidade particular", ou seja, os EVs pressupõem a apropriação de uma cultura particular para que o indivíduo possa viver, agir e dominar o meio. Para Durkheim (1963 apud GONÇALVES, 2004, p. 3), os EVs são as “[...] maneiras de agir, pensar e sentir". De maneira aparentemente mais simples, Rocher (1989, apud GONÇALVES, 2004, p. 3) definiu EV como “[...] as maneiras de viver", entendendo maneiras como sendo o ajuste de todas as dimensões do sujeito individual à coletividade social e ambiental, e viver implica a realização das vontades, desejos e anseios da pessoa.

Atualmente, o termo EV envolve todos os aspectos da ação e do pensamento humano (educação, recreio/lazer, nutrição, paz, justiça, trabalho, família, habitação, higiene, segurança, alimentação, recursos econômicos, recursos ambientais, tabagismo, alcoolismo, comportamentos de risco em relação às drogas ilícitas e às infecções sexualmente transmissíveis). Estes aspectos são tidos como elementos estruturantes e requisitos indispensáveis à obtenção do completo bem-estar físico, mental e social (GONÇALVES, 2004).

Parece ser consensual, entre os especialistas da área da saúde, que o EV, caracterizado por um conjunto de hábitos e comportamentos, adotado pelos indivíduos da sociedade moderna, tanto por jovens quanto por adultos, representa um dos principais responsáveis pelas alterações relacionadas ao perfil de morbimortalidade nas últimas décadas (BLAIR et al., 1996; CERVATO et al., 1997). Entre estes comportamentos, os níveis insuficientes de atividade física, os hábitos alimentares inadequados (consumo elevado de gorduras saturadas e um baixo consumo de frutas e verduras), o tabagismo, o consumo de bebidas alcoólicas, o sobrepeso/obesidade e os comportamentos sexuais de risco, têm recebido maior destaque (USDHHS, 2000).

Apesar de todas as evidências das atitudes que promovem um EV mais saudável e consequentemente seus benefícios, as pessoas, de um modo geral, possuem hábitos de vida bastante diferentes do que se considera saudável. De acordo com o Ministério da Saúde do Brasil, a prevalência de adultos jovens (19 a 35 anos) saudáveis, que são aqueles não fumantes, que praticam 
atividade física de forma regular e apresentam consumo adequado de frutas e hortaliças, estão em torno de 8,0\% da população (BRASIL, 2003). Os demais jovens brasileiros de 18 a 29 anos de idade relataram de dois a nenhum comportamento saudável, o que os tornam sujeitos com grandes possibilidades de adquirir doenças e agravos não transmissíveis à saúde em futuro próximo (BARRETO et al., 2009).

A atividade física e os hábitos alimentares são dois elementos do EV que desempenham um papel significativo na promoção da saúde e na prevenção de doenças (BLAIR et al., 1996). Além disso, outros elementos do EV são também importantes para a saúde e o bem-estar, tais como: evitar o uso de cigarros, possuir um bom relacionamento com a família e amigos, evitar o consumo de álcool, praticar o sexo seguro, controlar o estresse, além da necessidade de se ter uma visão otimista e positiva da vida (CSEP, 1998).

\subsection{Estilo de vida: como avaliar?}

De maneira geral, para avaliar o EV, sobretudo de universitários, são utilizados questionários, pois são de baixo custo, de fácil aplicação e permitem avaliar um grande número de pessoas, o que os torna muito apropriados para estudos epidemiológicos, pois proporcionam respostas rápidas para algo que exigiria muito tempo para ser avaliado de outra forma.

Um dos questionários utilizados é o 'Estilo de Vida Fantástico' que é um instrumento genérico que foi desenvolvido no Departamento de Medicina Familiar da Universidade McMaster, no Canadá, por Wilson e Ciliska (1984). O questionário tem por objetivo mensurar os principais elementos que caracterizam o EV adequado para a saúde. O instrumento consiste de um questionário padronizado com 25 questões fechadas que exploram 9 domínios sobre os componentes físicos, psicológicos e sociais do EV (ÃNEZ; REIS; PETROSKI, 2008).

Em 2008, Añez, Reis e Petroski (2008) realizaram a tradução e a validação do 'Estilo de Vida Fantástico' para a população brasileira. A tradução foi realizada pelos autores e submetida à apreciação de especialistas fluentes na língua inglesa. Para verificar a clareza, depois de traduzido, o questionário foi aplicado a 18 indivíduos, verificando com os mesmos se haveria alguma dificuldade em responder as perguntas. Na validação, a amostra não-probabilística foi constituída por 62 adultos jovens de ambos os sexos, recrutados por voluntariedade entre os estudantes de graduação e pós-graduação da Universidade Federal de Santa Catarina. Para verificação da estabilidade do instrumento, duas coletas de dados foram realizadas, avaliando sua consistência interna, reprodutibilidade e a validade de construto do instrumento. Para os autores, o questionário preencheu os critérios de consistência interna e de validade de construto, apresentando evidências de que é adequado para a avaliação do EV de adultos jovens brasileiros, recomendando sua utilização na atenção primária e em estudos epidemiológicos.

Outro instrumento utilizado para estimar o EV é o 'Questionário Saúde na Boa', desenvolvido por Nahas et al. (2007). O questionário é composto de cinco seções, cada qual organizada com questões estruturadas. A primeira seção inclui informações demográficas (sexo, idade, estado civil, trabalho, tipo de residência e cor da pele). A segunda e a terceira seções, objetos do presente estudo, incluem, respectivamente, informações sobre atividades físicas e comportamentos sedentários e sobre a frequência de consumo de alimentos selecionados. A quarta seção se refere ao controle do peso corporal e a quinta a comportamentos preventivos (NAHAS et al., 2007). No módulo de atividade física, o instrumento avalia o número de dias por semana (semana típica e últimos 7 dias) que o indivíduo pratica 60 minutos ou mais de atividades físicas de intensidade moderada ou vigorosa, sendo calculada a média desses escores. Para orientar o respondente sobre o que representa uma atividade física moderada ou intensa, há no questionário uma descrição e exemplos de atividades com este nível de intensidade. Na seção nutrição foram selecionados os seguintes alimentos ou grupos de alimentos: frutas, sucos naturais de frutas, verduras, saladas verdes, carne vermelha, embutidos, salgadinhos, doces, refrigerantes, leite e derivados e feijão com arroz. As questões versaram sobre a frequência de consumo semanal, não sendo incluídas perguntas sobre as quantidades consumidas em cada dia. 
Nahas, Barros e Francalacci (2000) desenvolveram outro questionário denominado 'Perfil do Estilo de Vida Individual'. O instrumento é subdividido em 15 itens dentre 5 fatores: nutrição, atividade física, comportamento preventivo, relacionamentos e controle do estresse. É utilizada uma escala de quatro pontos para responder aos itens: 0 (nunca), 1 (raramente), 2 (quase sempre) e 3 (sempre). Para desenvolver o questionário, os autores inspiraram-se no modelo do 'Pentáculo do Bem-Estar', que é uma demonstração gráfica dos resultados obtidos através do questionário do perfil do EV individual, sendo demonstrada em figura no formato do Pentáculo (estrela), com intuito de facilitar a visualização dos seguimentos abordados.

Os instrumentos que visam avaliar o EV, desenvolvidos e validados no Brasil, têm recebido grande aceitação entre os estudiosos da área. Em estudos que objetivam avaliar o EV de estudantes universitários brasileiros, os questionários 'Estilo de Vida Fantástico' e 'Perfil do Estilo de Vida Individual' vêm sendo os instrumentos mais utilizados para análise deste construto.

\subsection{Estilo de vida de estudantes universitários}

Grande parte do EV é estabelecido enquanto jovem, podendo influenciar na saúde e bemestar na vida adulta e na velhice. Para Silva et al. (2011), alguns fatores do EV podem ser introduzidos no cotidiano dos jovens no momento da transição do ensino médio para o superior, momento em que ocorre questionamento de valores, crenças e atitudes empregados pela família no processo de educação. Estes questionamentos podem refletir em um novo comportamento que, por consequência, pode determinar um estado de saúde de forma mais positiva ou negativa.

Refletindo sobre aspectos relacionados ao EV, reporta-se ao cotidiano da população universitária e da observação empírica da prática, aonde se evidenciam situações como: carga horária extensa em sala de aula e campo de estágio, sendo que as atividades complementares são desenvolvidas em outro período, geralmente no horário de refeições, à noite, finais de semana e feriados; uso abusivo de álcool e outras drogas; reduzido tempo de sono/repouso; hábito alimentar inadequado; não realização de atividade física regular; ansiedade/angústia constante pela cobrança do desempenho acadêmico; adaptação à outra cidade; afastamento temporário da família, namorado(a) e amigos(as) (SOARES; CAMPOS, 2008).

$\mathrm{O}$ álcool é a droga mais consumida entre os jovens, sendo um importante fator de risco para a adoção de outras condutas prejudiciais para a saúde. Entre estudantes americanos foi encontrada relação do consumo de álcool até a embriaguez com o consumo de cigarro, maconha, cocaína e outras drogas (JONES et al., 2001). Em outro estudo, Douglas et al. (1995) observaram que os universitários americanos apresentavam diversos fatores de risco, entre eles: consumo de maconha $(14,0 \%)$, consumo de cocaína $(14,4 \%)$ e alto percentual de atividade sexual $(86,1 \%)$. De acordo com os autores do estudo, estes achados servem para confirmar a importância do monitoramento de tais comportamentos.

No Reino Unido, a cada dois anos é realizado o estudo Sodexo University Lifestyle Survey (SODEXO, 2012). Esta pesquisa é desenvolvida com estudantes universitários de todo o país e tem como objetivo compreender quais as necessidades dos alunos, buscando oferecer um melhor serviço. Na sua última versão, em 2012, foram encontrados os seguintes resultados: em relação à forma como estes alunos se socializam, quase um terço dos estudantes $(31,0 \%)$ conversava na maioria das vezes dentro de seu próprio alojamento ou no de seus amigos. No domínio nutrição, menos da metade (43,0\%) afirmaram tomar café da manhã todos os dias, enquanto 12,0\% afirmaram que nunca o tomavam. Entre outras descobertas, o estudo constatou que 45,0\% dos avaliados não almoçavam pelo menos uma vez na semana e que a refeição mais regular foi o jantar (80,0\%). Não obstante, $81,0 \%$ disseram que faziam esforço para se alimentar de forma saudável. Dentre os avaliados, 51,0\% dos participantes disseram ter bebido menos de 10 unidades de álcool por semana (equivalente a cerca de 4,5 1 de cerveja), apenas $1,0 \%$ relatou que bebia mais de 41 unidades por semana (cerca de 201 ) e 6,0\% bebiam entre 21 e 40 unidades. Para a coleta dos dados, foram realizadas 2001 entrevistas online, através de um questionário que continha 32 perguntas e 
demorava em média 18 minutos para ser respondido (THE SODEXHO UNIVERSITY LIFESTYLE SURVEY, 2012).

$\mathrm{Na}$ Colômbia, Lema et al. (2009) realizaram um estudo com 587 estudantes de uma universidade privada (44,7\% homens e 55,3\% mulheres), com idades que variavam entre 16 e 30 anos, de diversos cursos das áreas de Humanas e Ciências Sociais, Engenharia e Ciências Econômicas e Administrativas. Os resultados indicaram que os universitários tinham um EV considerado saudável na maioria das dimensões, com exceção do aspecto exercício e atividade física, já que $77,0 \%$ dos participantes relataram que não realizavam algum tipo de exercício por pelo menos 30 minutos.

Faria (2012) realizou um estudo com 365 estudantes dos dois primeiros e dos dois últimos anos do Mestrado Integrado em Medicina da Faculdade de Ciências da Saúde da Universidade da Beira Interior, em Portugal. No estudo foram comparados os diferentes anos de estudo e avaliadas as associações entre as diferentes variáveis do EV. O sono e o estresse não apresentaram diferenças estatisticamente significativas entre os primeiros e os últimos anos, bem como, os estudantes no fim do curso apresentaram hábitos alimentares mais saudáveis. $\mathrm{O}$ domínio atividade física não apresentou diferenças entre os grupos, pois ambos não praticavam atividades físicas regularmente, e os níveis de experimentação de tabaco foram semelhantes em ambos os grupos. Quanto ao consumo de álcool, foi detectado maior número de consumidores no grupo de alunos dos primeiros anos. Foi utilizado um questionário desenvolvido especificamente para o estudo baseado no questionário 'Estilo de Vida Fantástico'.

$\mathrm{Na}$ Espanha foi realizado um estudo com 985 estudantes da Universidade de Vigo, no campus de Pontevedra, durante os anos letivos de 2009 e 2010 (VARELA-MATO et al., 2012). Dentre os participantes, $67,4 \%$ eram mulheres e pertenciam aos cursos da área de saúde, educação e demais cursos (Publicidade e Relações Públicas, Belas Artes, Gestão Pública, Administração, Comunicação Audiovisual e Engenharia Florestal). O nível de atividade física foi avaliado por meio da versão em espanhol do 'Physical Activity Questionnaire Internacional' (IPAQ), versão curta. A versão em espanhol do 'Teste de Atitudes Alimentares' (EAT-40) foi usada para detectar distúrbios no comportamento alimentar e atitudes em relação à nutrição. Em relação ao uso de tabaco e consumo de substâncias tóxicas, foi utilizado um questionário elaborado especificamente para este fim em populações jovens. Os resultados de Varela-Mato et al. (2012) indicaram que apenas 27,4\% dos alunos poderiam ser considerados como suficientemente ativos, enquanto que $14,9 \%$ deles sofriam de transtornos alimentares. O consumo diário de álcool encontrado foi baixo (1,5\%), mas a porcentagem de consumidores habituais foi elevada (77,0\%). Com relação ao consumo de tabaco, cerca de um terço dos alunos, de ambos os sexos, alegaram serem fumantes habituais. Proporção semelhante foi encontrada entre os que admitiram ter usado substâncias ilícitas (VARELA-MATO et al., 2012).

No Brasil, estudos epidemiológicos e de pesquisa com delineamentos metodológicos longitudinais são escassos, a maioria dos estudos sobre o EV de universitários vem sendo realizada em pequenos grupos populacionais e com delineamento transversal (SILVA, et al., 2011; SILVA, et al., 2012; CONCEIÇÃO; DUZZIONI, 2008).

Marinho (2002) realizou estudo com 357 estudantes da Universidade do Planalto Catarinense, localizada em Lages, Santa Catarina. Os participantes eram de ambos os sexos (40,4\% homens e 59,6\% mulheres) e de áreas de conhecimento diferenciadas (Saúde, Exatas e Tecnológicas, Econômico-Jurídica e Ciências Humanas, Letras e Artes). O instrumento utilizado foi o questionário 'Pentáculo do Bem-Estar'. A análise dos dados indicou que 78,4\% dos participantes não eram fumantes, 46,6\% relataram não estar satisfeitos com seu peso corporal e 76,1\% indicaram percepção de saúde positiva. $\mathrm{O}$ estudo indicou, ainda, que a amostra era pouco ativa fisicamente e com alimentação rica em gorduras, refrigerantes e doces, porém, com baixo consumo de álcool e fumo.

Em pesquisa realizada por Joia (2010), em Barreiras, Bahia, foram avaliados 257 estudantes da Faculdade São Francisco, com idades que variavam entre 20 e 40 anos, dos cursos de Fisioterapia, Enfermagem, Educação Física, Psicologia, Agronomia, Direito, Ciências Contáveis, 
Administração, Comunicação Social e Pedagogia. Através do 'Questionário Estilo de Vida Individual', o autor observou que quase a metade dos entrevistados $(49,0 \%)$ relataram que na sua alimentação diária apenas às vezes incluíam ao menos 5 porções de frutas, 45,5\% dos entrevistados não realizavam ao menos 30 minutos de atividades físicas moderas e/ou intensas, 38,9\% não caminhavam no seu dia a dia e 37,7\% utilizavam preferencialmente o elevador ao invés de escadas. A maioria dos universitários $(75,5 \%)$ cultivava amigos e estava satisfeita com seus relacionamentos, bem como, $34,2 \%$ reservavam tempo durante o dia para relaxar.

Utilizando o questionário 'Estilo de Vida Fantástico', Silva et al. (2012) realizaram um estudo com 217 universitários do curso de Educação Física de uma universidade pública do estado de Sergipe. Entre os participantes da pesquisa, 119 eram do sexo masculino e em sua maioria com idade inferior a 20 anos. Em relação aos domínios do EV, constatou-se que 15,2\% apresentaram uma relação com a(os) família/amigos inadequada, 57,4\% não atenderam as recomendações para atividade física, 41,9\% reportaram uma alimentação inadequada, 1,8\% fazia uso de tabaco e/ou outros tóxicos, $8,8 \%$ ingeriam bebidas alcoólicas em excesso, 12,5\% apresentaram problemas com sono e estresse, $10,0 \%$ tinham mau comportamento ao dirigir, 50,7\% sentiam-se frequentemente com raiva e hostis, $22,1 \%$ tinham problemas de introspecção e $14,3 \%$ declararam insatisfação com o(a) trabalho/função que exerciam.

Silva et al. (2011) desenvolveram uma pesquisa com 685 universitários da Universidade Federal de Santa Catarina, utilizando o questionário 'Estilo de Vida Fantástico'. Entre os participantes, 410 eram do sexo masculino, com média de idade de 20,4 anos. Os autores investigaram a associação do sobrepeso e de variáveis sociodemográficas com o EV. Os achados demonstraram que $5,1 \%$ dos avaliados apresentaram um EV considerado pouco saudável, e que o risco para sobrepeso foi maior entre universitários que relataram estar com mais de dois quilos acima do peso considerado saudável, com os que se sentiam tensos e desapontados, com os que não possuíam uma dieta balanceada, que ingeriam bebidas com cafeína mais de duas vezes por dia e com uma média de ingestão de álcool por semana superior a sete doses ( 1 dose $=1$ lata de cerveja $340 \mathrm{ml}$ ou 1 copo de vinho - $142 \mathrm{ml}$ ou 1 copo pequeno de destilado - $42 \mathrm{ml}$ ).

Em investigação realizada por Conceição e Duzzioni (2008), com 137 acadêmicos de 17 a 38 anos de idade do curso de Educação Física, em Criciúma, Santa Catarina, através do questionário 'Pentáculo do Bem Estar', foram encontrados os seguintes resultados: 22,7\% dos universitários não incluíam na sua alimentação diária 5 porções de frutas e verduras, 18,7\% não realizavam ao menos 30 minutos de atividade física de intensidade moderada a intensa. Em relação ao uso de álcool e tabaco, notou-se um consumo elevado $(22,4 \%$ responderam que fumavam ou ingeriam bebidas alcoólicas sem moderação). Ainda, $38,1 \%$ dos investigados não usavam sempre o cinto de segurança, 32,8\% não estavam satisfeitos com seus relacionamentos e 52,2\% não reservavam tempo (ao menos cinco minutos) todos os dias para relaxar.

Utilizando também o 'Pentáculo do Bem Estar', Santos e Alves (2009) realizaram estudo, com 523 acadêmicos dos cursos de Educação Física, Psicologia, Fonoaudiologia, Engenharia Florestal, Engenharia Ambiental e Turismo da Universidade Estadual do Centro-Oeste, Campus de Irati, Paraná. Observou-se que 71,5\% dos universitários apresentaram ao menos um comportamento considerado inadequado, 69,0\% dos estudantes não praticavam regularmente algum tipo de atividade física por no mínimo 30 minutos por dia e 55,0\% não realizavam exercícios de força e alongamento ao menos três vezes por semana. Entre os participantes, 7,8\% afirmaram não respeitar as normas de trânsito, 1,1\% alegaram nunca cultivar amigos, 30,0\% afirmaram não reservar ao menos cinco minutos do seu dia para relaxar e/ou meditar e 51,2\% dos estudantes não conseguiam manter uma discussão sem se alterar.

Ainda que a quantidade de estudos tenha aumentado nos últimos anos, os estudos acerca deste construto ainda são escassos e por isso ainda há muito que investigar sobre o EV e comportamentos relacionados à saúde de estudantes universitários. Há evidências, em diversos países, de que estratégias envolvendo as mudanças de comportamentos e, portanto no EV, produzem resultados positivos e devem ser implementadas rotineiramente nos níveis pessoal, institucional e comunitário (YUSUF, 2002; MARMOT, 2001; VARTIAINEN et al., 2000; PEKKA 
et al., 2002). Para Vieira et al. (2002), as modificações quanto aos padrões alimentares, prática de atividade física, uso do cigarro e ingestão de álcool, somadas a situações próprias da adolescência, como intensas alterações biológicas, instabilidade psicossocial e falta de comportamento preventivo, podem tornar os universitários um grupo vulnerável a riscos significativos com relação a sua saúde. A população universitária tem padrões típicos de consumo que diferem do restante da população, sendo necessário o desenvolvimento de programas de promoção da saúde específicos e integrados com intervenções direcionadas para os múltiplos fatores de risco.

\section{Considerações finais}

$\mathrm{O}$ aumento no interesse pelo tema EV está relacionado à constante preocupação com a melhoria das condições de vida da população através de ações que possibilitem a adesão aos comportamentos saudáveis. Os achados do presente estudo indicaram relevante avanço do conhecimento sobre o EV durante os últimos anos, com evolução interessante das definições e dos instrumentos desenvolvidos para sua avaliação. No entanto, observa-se escassez de estudos epidemiológicos, bem como, de pesquisas com delineamentos metodológicos longitudinais, que são fundamentais para o estabelecimento de relações de causa e efeito entre exposição e desfecho.

Os resultados das investigações revisadas no presente estudo demonstraram que grande parte dos estudantes universitários tem adotado um EV pouco saudável, especialmente no que se refere ao consumo de álcool, tabaco e outras drogas, prática insuficiente de atividades físicas e hábitos alimentares inadequados. Neste sentido, observa-se a necessidade da elaboração e implantação de políticas públicas de promoção da saúde dentro da própria Universidade, com vistas à melhoria da saúde e da qualidade de vida dos estudantes universitários.

\section{Referências}

AÑEZ, C. R. R.; REIS, R. S.; PETROSKI, E. L. Versão brasileira do questionário "estilo de vida fantástico": tradução e validação para adultos jovens. Arquivos Brasileiros de Cardiologia, São Paulo, v. 91, n. 2, p. 102-109, 2008.

ASTRAND, P. O. Porque fazer exercício? Revista Brasileira de Ciência e Movimento, Brasília, v. 13 , n. 4 , p. 39-56, 1993.

BARRETO, S. M.; PASSOS, V. M. A.; GIATTI, L. Comportamento saudável entre adultos jovens no Brasil. Revista de Saúde Pública, São Paulo, v. 43, n. 2, p. 9-17, 2009. rrossef

BLAIR, S. N.; HORTON, E.; LEON, A. S.; DRINKWATER, B. L.; DISHMAN, R. K.; MACKEY, M.; KIENHOLZ, M. L. Physical activity, nutrition, and chronic disease. Medicine and Science in Sports and Exercise, Madison, v. 28, n. 3, p. 335-349, 1996.

BRASIL. Ministério da Saúde. Instituto Nacional de Câncer. Programa nacional de controle do tabagismo e outros fatores de risco de câncer-modelo lógico e avaliação. 2. ed. Brasília: Ministério da Saúde; Instituto Nacional do Câncer, 2003.

CANADIAN SOCIETY FOR EXERCISE PHYSIOLOGY. The Canadian physical activity, fitness and lifestyle appraisal: CSEP's guide to health active living. 2. ed. Ottawa: CSEF, 1998.

CERVATO, A. M.; MAZZILlI, R. N.; MARTINS, I. S.; MARUCCI, M. F. N. Dieta habitual e fatores de risco para doenças cardiovasculares. Revista de Saúde Pública, São Paulo, v. 31, n. 3, p. 227-235, 1997. Crossef 
CONCEIÇÃO, V. J. S.; DUZZIONI, F. I. Estilo de vida de universitários: um estudo descritivo com acadêmicos do curso de Educação Física. Lecturas - Educación Física y Deportes, Buenos Aires, ano 13, n. 124, set. 2008. Disponível em: <http://www.efdeportes.com/efd124/estilo-de-vidade-universitarios-um-estudo-descritivo-do-curso-de-educacao-fisica.htm>. Acesso em: 02 set. 2013.

DOUGLAS, K. A.; COLLINS J. L.; WARREN, C.; KANN, L.; GOLD, R., CLAYTON, S., ROSS, J. G.; KOLBE, L. J. Results from the 1995 National College Health risk behavior survey. Journal of American College Health, Carbondale, v. 46, n. 2, p. 55-67, 1997. crossef

FARIA, D. L. S. Estudo comparativo dos estilos de vida dos estudantes de medicina da Universidade da Beira Interior no início e no final do curso. 2012. 38 f. Dissertação (Mestrado em Medicina) - Universidade da Beira Interior, Portugal, 2012.

GONÇALVES, A. Diferenças de estilos de vida entre populações jovens de meio rural (Boticas) e de meio urbano (Braga): análise de concepções, de valores e de práticas. 2004. $162 \mathrm{f}$. Dissertação (Mestrado em Estudos da Criança-Promoção da Saúde e do Meio Ambiente) Universidade do Minho-Braga, Portugal, 2004.

HEYWARD, V. H. Advanced fitness assessment e exercise prescription. 3. ed. Champaign: Human Kinetics, 1998.

JOIA, L. C. Perfil do estilo de vida individual entre estudantes universitários. Revista Movimenta, Goiânia, v. 3, n. 1, p. 16-23, 2010.

JONES, S. E.; OELTMANN, J.; WILSON, T. W.; BRENER, N. D.; HILL, C. V. Binge drinking among undergraduate college students in the United States: implications for other substance use. Journal of American College Health, Carbondale, v. 50, n. 1, p. 33-38, 2001. rossef

LEMA, L. F.; SALAZAR, I. C.; VARELA, M. T.; TAMAYO, J. A.; RUBIO, A.; BOTERO, A. Comportamiento y salud de los jóvenes universitarios: satisfacción con el estilo de vida. Pensamiento Psicológico, Cali, v. 5, n. 12, p. 71-88, 2009.

MARINHO, C. S. Estilo de vida e indicadores de saúde de estudantes universitários da Uniplac. 2002. 76f. Dissertação (Mestrado em Educação Física) - Centro de Desportos Programa de Pós-Graduação em Educação Física, Universidade Federal de Santa Catarina, Florianópolis, 2002.

MARMOT, M. Economic and social determinants of disease. Bullettin of the World Health Organization, Geneva, v. 79, p. 988-989, 2001.

MOTA, J.; DUARTE, JA. Estilo de vida activo e saúde. Boletim da Sociedade Portuguesa de Educação Física, Lisboa, n. 17-18, p. 47-51, 1999.

NAHAS, M. V. N; BARROS, M. V. G, FLORINDO, A. A; FARIAS, J. C. J; HALLAL, P. C.; KONRAD, L.; BARROS, S. S. H.; ASSIS, M. A. A. Reprodutibilidade e validade do questionário saúde na boa para avaliar atividade física e hábitos alimentares em escolares do ensino médio. Revista Brasileira de Atividade Física e Saúde, Pelotas, v. 12, n. 3, p. 12-20, 2007.

NAHAS, M. V.; BARROS, M. V. G.; FRANCALACCI, V. L. O Pentáculo do Bem-Estar: base conceitual para avaliação do estilo de vida de indivíduos ou grupos. Revista Brasileira de Atividade Física e Saúde, Pelotas, v. 5, n. 2, p. 48-59, 2000. 
PEKKA, P.; PIRJO, P.; ULLA, U. Influencing public nutrition for non-communicable disease prevention: from community intervention to national programme - experiences from Finland. Public Health and Nutrition, Londres, v. 5, n. 1(A), p. 245-251, 2002.

RICHARDSON, C. R.; KRISKA, A. M.; LANTZ, P. M.; HAYWARD, R. A. Physical activity and mortality across cardiovascular disease risk groups. Medicine and Science in Sports and Exercise, Madison, v. 36, n. 11, p. 1923-1929, 2004. crossef

SANTOS, J. F. S.; ALVES, V. S. Perfil do estilo de vida relacionado à saúde dos acadêmicos da Unicentro, Campus Irati, PR. Lecturas - Educación Física y Deportes, Buenos Aires, ano 13, n. 129, 2009. Disponível em: <http://www.efdeportes.com/efd129/perfil-do-estilo-de-vidarelacionado-a-saude-dos-academicos.htm>. Acesso em: 02 jun. 2013.

SILVA, D. A. S.; QUADROS, T. M. B.; GORDIA, A. P.; PETROSKI, E. L. Associação do sobrepeso com variáveis sócio-demográficas e estilo de vida em universitários. Revista Ciência e Saúde Coletiva, Manguinhos, v. 16, n. 11, p. 4473-4479, 2011.

SILVA, D. A. S.; PEREIRA, I. M. M.; ALMEIDA, M. B.; SILVA, R. J. S.; OLIVEIRA, A. C. C. Estilo de vida de acadêmicos de educação física de uma universidade pública do estado de Sergipe, Brasil. Revista Brasileira de Ciências do Esporte, Porto Alegre, v. 34, n. 1, p. 53-67, 2012. crossef

SOARES, R. D. O. P. S.; CAMPOS, L. F. Estilo de vida dos estudantes de enfermagem de uma universidade do interior de Minas Gerais. Cogitare Enfermagem, Curitiba, v. 13, n. 2, p. 227-234, 2008.

SODEXO. The Sodexho University Lifestyle Survey, 2012. Sodexo and the Times Higher Education Supplement. Disponível em: 〈http://avanconspourluniversite.sodexo.fr/documents/student-survey-uk.pdf $>$. Acesso em: $16 \mathrm{dez}$. 2013.

US DEPARTMENT OF HEALTH AND HUMAN SERVICES. Center for Disease Control and Prevention, 2000. Disponível em: <www.cdc.gov/needphp/dash/yrbs/yrbsaag.htm>. Acesso em: 14 jan. 2013.

VARELA-MATO, V.; CANCELA J. M.; AYAN, C.; MARTIN, V.; MOLINA, A. Lifestyle and health among Spanish university students: differences by gender and academic discipline. International Journal of Environmental Research and Public Health, Basel, v. 9, n. 8, p. 27282741, 2012. crossef

VARTIAINEN, E.; JOUSILAHTI, P.; ALFTHAN, G.; SUNDVALL, J.; PIETINEN, P.; PUSKA, P. Cardiovascular risk factor changes in Finland, 1972-1997. International Journal of Epidemiology, Oxford, v. 29, p. 49-56, 2000. Crossef

VIEIRA, V. C. R.; PRIORE, S. E.; RIBEIRO, S. M. R.; FRANCESCHINI, S. C. C.; ALMEIDA, L. P. Perfil socioeconômico, nutricional e de saúde de adolescentes recém-ingressos em uma universidade pública brasileira. Revista de Nutrição, Campinas, v. 15, n. 3, p. 273-282, 2002.

VILARTA, R.; GONÇALVES, A. Condições de vida, modo de vida e estilo de vida. In: GONÇALVES, A.; VILARTA, R. (Ed.) Qualidade de vida e atividade física. Barueri: Manole, 2004. 
WILSON, D. M.; CILISKA, D. Lifestyle assessment: testing the FANTASTIC instrument. Canadian Family Physician, Mississauga, v. 30, p. 1863-1866, 1984.

WORLD HEALTH ORGANIZATION. Health Promotion Glossary. 1998. Disponível em: http://www.who.int/healthpromotion/about/HPR\%20Glossary\%201998.pdf. Acesso em: 14 jan. 2013.

YUSUF, S.; PITT, B. A Lifetime of prevention: the case of heart failure. Circulation, Dallas, v. 106, p. 2997-2998, 2002. Crossef 